Felix Wilhelm Siebert, Fares Lian Wallis

\title{
How speed and visibility influence preferred headway distances in highly automated driving
}

Journal article | Accepted manuscript (Postprint)

This version is available at http://dx.doi.org/10.14279/depositonce-8761

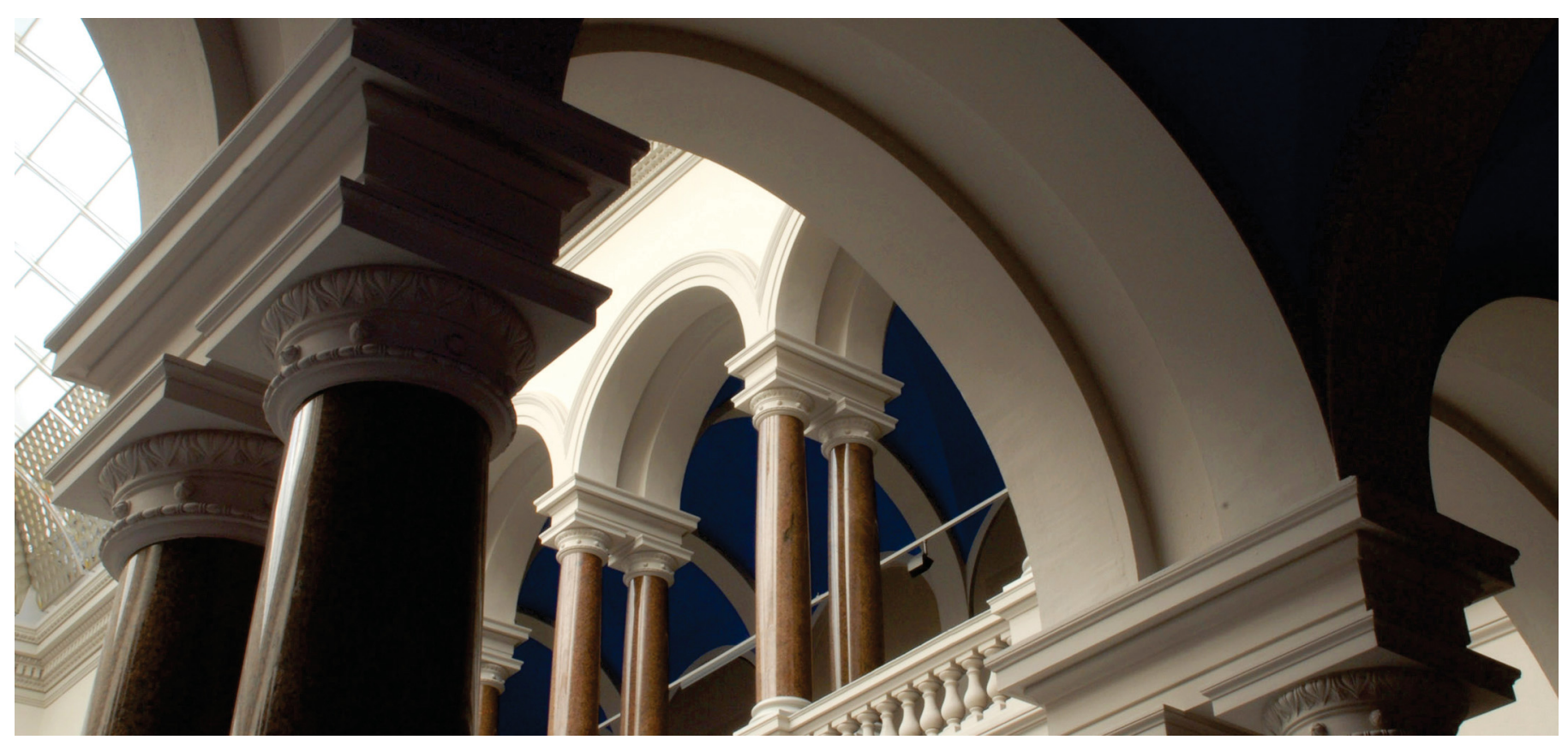

Siebert, F. W., \& Wallis, F. L. (2019). How speed and visibility influence preferred headway distances in highly automated driving. Transportation Research Part F: Traffic Psychology and Behaviour, 64, 485494. https://doi.org/10.1016/j.trf.2019.06.009 
This is the Accepted Manuscript of the following article published by Elsevier in Transportation Research Part F: Traffic Psychology and Behaviour [6. July 2019]:

9 Siebert, F. W., \& Wallis, F.L. (2019). How speed and visibility influence preferred headway distances 10 in highly automated driving. Transportation Research Part F: Traffic Psychology and Behaviour, 64, 485-594. https://doi.org/10.1016/j.trf.2019.06.009 This manuscript is not the copy of record and may not exactly replicate the final, authoritative version of the article. International License, http://creativecommons.org/licenses/by-nc-nd/4.0/. 

driving

Felix Wilhelm Siebert ${ }^{\mathrm{a}} \&$ Fares Lian Wallis ${ }^{\mathrm{b}}$

Corresponding Author: Felix Wilhelm Siebert

27

${ }^{\text {a }}$ Chair of Work, Engineering \& Organizational Psychology

Department of Psychology and Ergonomics

30

Technische Universität Berlin

31 Marchstraße 12

$32 \quad 10587$ Berlin

33 Germany

${ }^{\mathrm{b}}$ Chair of Engineering Psychology and Applied Cognitive Research

Technische Universität Dresden

36 Dresden

37 Germany

E-mail: felix.siebert@tu-berlin.de

E-mail: fares.wallis@mailbox.tu-dresden.de

40 


\section{Abstract}

While the introduction of highly automated vehicles promises lower accident numbers, a main requirement for wide use of these vehicles will be the acceptance by drivers. In this study a crucial variable for the acceptance of highly automated vehicles, the vehicle to vehicle distance expressed in time headway, was researched in a driving simulator. Research has shown that time headway distances, perceived as comfortable in self-driving and assisted driving with adaptive cruise control, remain constant over a range of different speeds. This study aims to test these findings for highly automated driving. Since time headway is perceived visually, the driving situation was varied to investigate the influence of visibility on the subjective comfort of the driver in a highly automated driving situation. In a withinsubject design, drivers followed a passenger car in clear weather conditions, the same passenger car in fog which occluded parts of the traffic environment, as well as a truck that occluded the lane ahead, also in clear weather condition. Subjective comfort of drivers in each condition was rated with a haptic rating lever.

Results suggest that comfortable time headway following distances in highly automated driving are not constant over different speeds, but that these distances decrease with increasing speed. Reduced visibility generally led to a shift in comfortable following distances towards larger headways. These results have implications for the introduction of highly automated vehicles and their time headway adjustments, which will need to be adaptive to speed and visibility in the road environment.

\section{Introduction}

Past research suggests that time headway is a variable held constant by individual drivers in self-driving (Siebert, Oehl, \& Pfister, 2014; Siebert, Oehl, Bersch, \& Pfister, 2017; Van Winsum \& Heino, 1996), and the individual choice of time headway has been related to the drivers' awareness of risk and comfort (Lewis-Evans, De Waard, \& Brookhuis, 2010). However, there has been comparatively little research on the influence of longitudinal vehicle to vehicle distances of highly automated vehicles on the subjective experience of drivers, with a small number of studies pointing to the importance of time headway adjustments in highly automated driving (Bellem, Schönenberg, Krems, \& Schrauf, 2016; De Waard, Van der Hulst, Hoedemaeker, \& Brookhuis, 1999). Since drivers will not be able to regulate their following distance in highly automated driving as freely as in self-driving, it is important to understand 
how time headway distances need to be adjusted for highly automated driving, without drivers feeling uncomfortable. Therefore, this study tested how results of constant time headway following from self- and assisted driving translate to highly automated driving. A general preference for constant time headway following in highly automated driving would imply that the complete secession of control by the driver of the car does not alter the effect of preferred constant time headways found in self- and assisted-driving. In turn, this would allow car manufacturers to program highly automated vehicles to follow at a constant time headway over a broad speed range.

Another goal of this study was to investigate the influence of different visibility conditions on preferred following distances in highly automated driving. Since time headway is the result of a visual estimation of the vehicle to vehicle distance divided by an estimation of the vehicle speed, the accuracy of an individual's time headway estimation depends on the visibility condition. Effects of changing following distances under adverse visibility on car following have been studied for self-driving, and we hope to extend this research to highly automated driving.

\subsection{Constant time headway following}

A large number of studies have found that drivers follow other vehicles with a constant time headway at different speeds, and prefer constant time headway following to non-constant following when presented with a number of time headways at different speeds (Ayres, Li, Schleuning, \& Young, 2001; Siebert et al., 2014, 2017; Taieb-Maimon \& Shinar, 2001; Van Winsum \& Heino, 1996). Siebert et al. (2014, 2017) researched car following preferences for the use of adaptive cruise control and found stable individual time headway preferences. Most preferred headways in self- and assisted-driving are found in the range of one to two seconds in simulated as well as in real-life driving, although preferred time headways of individual drivers differ. In all earlier studies on the relation between the subjective experience of drivers and time headway, drivers either had complete control over the vehicle (Ayres et al., 2001; Taieb-Maimon \& Shinar, 2001; Van Winsum \& Heino, 1996), or were actively controlling the steering wheel in studies where an adaptive cruise control system was implemented (Siebert et al., 2014, 2017). It is therefore unclear how the complete absence of active control over the vehicle in highly automated driving influences the subjective experience of time headways. Researchers have hypothesized that speed influences the subjective experience of 
drivers in in highly automated driving differently than in self- or assisted driving, due to a lack of immediate controllability of the driving situation (De Vos, Theeuwes, Hoekstra, \& Coëmet, 1997; Telpaz, et al., 2018).

\subsection{Driving in fog and behind larger vehicles}

Van Winsum (1999) postulates in his mathematical model of human car following that a reduced visibility in the driving environment due to fog or rain should in theory lead to an increase in time headway "as an increase of the safety margin to compensate for later detections of decelerations of lead vehicles" (p. 209). However, researchers have found conflicting results for following behavior during fog. While in some studies drivers increase their time headway when visibility is reduced (Van der Hulst, Rothengatter, Meijman, 1998) and their perceived risk is increased (Saffarian, Happee, De Winter, 2012), in other studies drivers follow closer when the visibility is reduced due to fog (Al-Ghamdi, 2007).

Researchers have also found interindividual differences when driving in heavy fog (visibility limit of $41 \mathrm{~m}$ ), with some drivers reducing time headway to within the visibility range of the lead vehicle, and other drivers increasing their time headway, thereby losing sight of the lead vehicle (Broughton, Switzer, and Scott, 2007).

Apart from reduced visibility of the driving environment due to weather, forward visibility can also be reduced when following large vehicles such as trucks or busses. For following larger vehicles there is no clear effect on following distances compared to following normal sized vehicles in self-driving. Studies have found increased time headways (Green \& Yoo, 1999; Wasielewski, 1981), decreased time headways (Brackstone, Waterson, \& McDonald, 2009; Sayer, Mefford, \& Huang, 2000), and increasing as well as decreasing time headways depending on driving speed (Duan, Li, \& Salvendy, 2013). Since this study is the first to compare following distances during clear and reduced visibility in highly automated driving, we base our hypotheses on Van Winsum's mathematical model of human car following (1999), and assume that a decrease in visibility will necessitate and increase in time headway distances. 


\subsection{Using a haptic lever for feedback on subjective experience in driving}

Different subjective variables have been used as dependent variables when participants are asked to rate their subjective experience of different time headways. Earlier studies have shown that subjective variables highly correlate with each other when time headways are rated (Lewis-Evans et al., 2010; Siebert et al., 2014). In this study, comfort was chosen as the dependent variable because it can be described in a positive and negative valence by the words comfort (German: angenehm) and discomfort (German: unangenehm). Due to translation imprecision, the German terms could also be translated as pleasant and unpleasant. Furthermore, a bi-directional haptic lever was used instead of single items in a likert-scale format used by Lewis-Evans et al. (2010) and Siebert et al. (2014). An advantage of this method is the simultaneous evaluation of the vehicle to vehicle distance, compared to a retrospective rating by a subsequent questionnaire. Additionally, the lever allows the participants to focus on the leading vehicle while rating the vehicle to vehicle distance since the lever can be adjusted without looking at it. The type of lever used in this study has been positively evaluated for linearity of ratings (Vehrs, 1986). A study by Charlton, Starkey, Perrone, and Isler (2014) showed that participants are able to concurrently rate the risk of a traffic situation by using a haptic risk-meter, similar in function to the lever used in this study.

\subsection{Goals of this study}

In this driving simulator study, the forward visibility of drivers in a highly automated vehicle was systematically varied at different speeds. To assess the impact of different time headways and reduced visibility on the subjective experience of the participants, drivers indicated their subjective level of comfort by moving a bi-directional haptic lever with their right hand. Participants were then presented with different vehicle to vehicle distances and the lever position was recorded continuously for these different distances.

We expected that in highly automated driving (1) speed does influence the comfort ratings for specific time headways and (2) reduced visibility reduces subjective comfort ratings for distances when compared to the clear visibility condition. 


\section{Method}

\subsection{Participants}

Thirty-nine participants took part in this study. Due to technical difficulties with the scaling lever, 4 participants were excluded from the analysis. All results reported in this paper are based on the sample of the 35 participants where no technical difficulties occurred. Of these 35 participants, 17 were female and 18 were male. Participants had a mean age of $M=22.46$ years $(S D=5.84)$. All participants were in possession of a valid driver's license, that they had acquired an average of $M=4.96$ years $(S D=5.86)$ before the study. On average, participants estimated to drive $M=8820.57$ kilometers per year $(S D=18902.6)$ with a minimum of 20 and a maximum of 100000 kilometers. The average accumulated driving experience of the participants was approximately 108,000 kilometers. About one third of the participants owned a car, and more than $50 \%$ of the participants used their own or another car at least once a week. Thirty-four participants were right-handed, with one participants being ambidextrous. Participants were recruited from the student body of the Leuphana University Lüneburg as a convenient sample. For their participation, participants were given "study-subject hours" that they have to acquire during their time at the university.

\subsection{Experimental design}

In this experiment, visibility was varied threefold (clear vs. truck vs. fog), speed was varied threefold $(50 \mathrm{~km} / \mathrm{h}$ vs. $100 \mathrm{~km} / \mathrm{h}$ vs. $150 \mathrm{~km} / \mathrm{h})$, and time headway was varied tenfold $(0.5 \mathrm{vs}$. 1.0 vs. 1.25 vs. 1.5 vs. 1.75 vs. 2.0 vs. 2.5 vs. 3.0 vs. 3.5 vs. 4.0 ). Two extra time headway increments (1.25 and 1.75 seconds) were added to more finely represent typical time headways found in earlier studies (Siebert et al., 2014, 2017). The resulting 90 experimental conditions were grouped in 9 blocks, each block consisting of a randomized order of ten time headways for the same visibility and speed. These 9 blocks were then randomly presented to participants. All participants were presented with the 90 experimental conditions in a withinsubject design.

Each experimental condition lasted 10 seconds, and each experimental block lasted about 120 seconds. There were short pauses of about 2-3 seconds between the conditions within each block, and longer pauses of 20-30 seconds between blocks, when a new block was loaded into the driving simulation software. 


\subsection{Driving simulator and driving environment}

206

207

208

209

210

211

212

213

214

215

216

217

218

219

220

221

222

223

224

225

226

227

228

229

230

231

232

233

The study was conducted at the Leuphana University Lüneburg in a fixed-base driving simulator cabin resembling a Volkswagen Golf 4 GTI with automatic transmission. To simulate the driving environment, SCANeR Studio Driving Simulation Software version 1.4 from Oktal was used. The driving environment was projected onto three screens in front of the simulator cabin for a total resolution of $3072 \times 768$ pixels with three video projectors. Each single screen had a size of $1.4 \times 1.4$ meters. The outer screens were positioned at an angle of $120^{\circ}$ to the center screen. The driver seat was positioned 2 meters from the center screen, resulting in a horizontal field of view of approximately $110^{\circ}$ and a vertical field of view of approximately $30^{\circ}$. The physical and simulated eye height of the participants was $1.25 \mathrm{~m}$. The simulated car model was a compact car, a Citroën $\mathrm{C} 4$. The speedometer of the simulator was inactive during the experiment. Simulation data were saved with a frequency of $20 \mathrm{~Hz}$.

Three driving environments were programmed for this study, with each environment representing a road type where a speed of 50,100 , or $150 \mathrm{~km} / \mathrm{h}$ could be expected. The $50 \mathrm{~km} / \mathrm{h}$ driving environment resembled an inner city road with one lane in each direction. The $100 \mathrm{~km} / \mathrm{h}$ driving environment was modelled after a German rural road, with two lanes in each direction, with opposing lanes divided by a solid line. The $150 \mathrm{~km} / \mathrm{h}$ condition was modelled after a German “Autobahn”, a highway road where the advised speed is $130 \mathrm{~km} / \mathrm{h}$, but generally there is no enforced speed limit. In this condition there were three lanes in each direction, and opposing traffic was separated by a guard railing. Each environment had only minimal road curvature, no slope, and sparse oncoming traffic. There were no side-streets in any of the road environments and there was no cross traffic by pedestrians. Road side buildings and trees had a minimum distance of 20 meters to the side of the road. The participant's vehicle and the lead vehicle always drove in the right-most lane.

Screenshots of the three visibility conditions are shown in Figure 1. The lead vehicle in the clear condition was a compact car, the lead vehicle in the truck condition was a truck, and the lead vehicle in the fog condition was the same compact car as in the clear condition. The fog in the fog condition was set to a range of $200 \mathrm{~m}$, resulting in light fog with a visibility limit of $200 \mathrm{~m}$. 


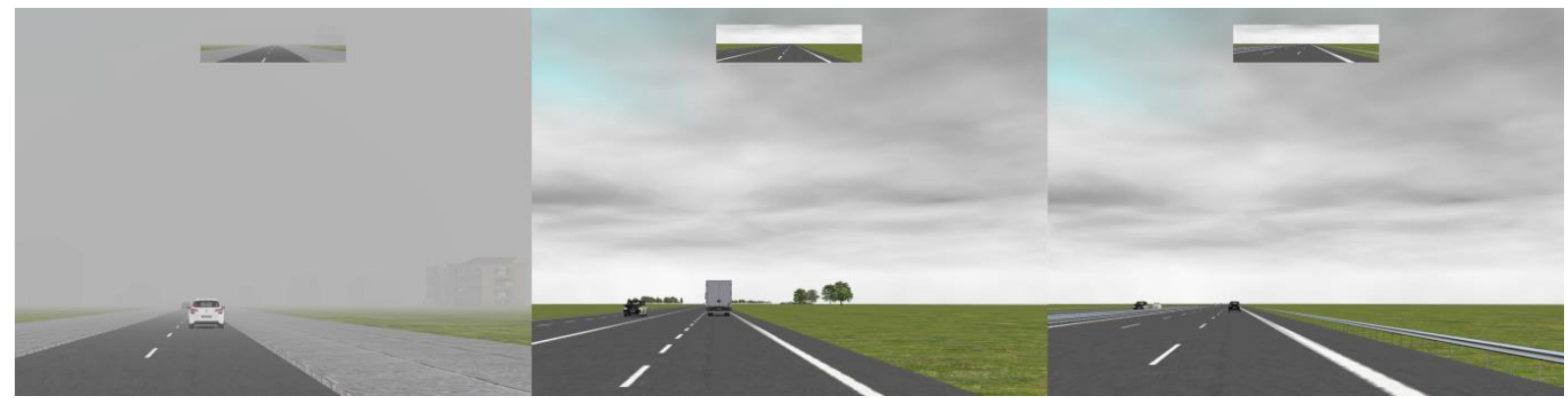

Figure 1. Screenshots of the center projection of the three visibility and three speed conditions for a time headway of 2 seconds: fog \& $50 \mathrm{~km} / \mathrm{h}$ (left), truck \& 100km $/ \mathrm{h}$ (middle), clear $\&$ $150 \mathrm{~km} / \mathrm{h}$ (right).

\subsection{Procedure}

After participants arrived at the simulator, they filled out a short demographic questionnaire and were then seated in the driver's seat of the simulator. The experimenter then explained the use of the simulator, and participants' task in the experiment. The instruction for using the rating lever was as follows (translated from German):

"Today you will be shown multiple driving situations in the driving simulator. During these situations, you do not need to control the car, as the car drives by itself. You do not need to steer, brake, or accelerate. Next to you there is a lever that can be moved in two directions, to the front and to the back. You will feel a light resistance that tries to automatically move the lever to a middle position. The lever position at the maximum front position represents "uncomfortable" (German "unangenehm"), the middle lever position represents "neutral" (German "neutral"), and the maximum back position is "comfortable" (German "angenehm"). Now take the lever into your hand and familiarize yourself with it by moving it to the front and the back multiple times. Now try some lever positions without looking at the lever. In the following you will see multiple consecutive driving situations. Please indicate the intensity of your feelings toward the distance to the lead vehicle, by adjusting the lever between "comfortable", "neutral", and "uncomfortable" and keeping the lever in this position for the whole driving situation. Please only rate the distance to the lead vehicle and not the other traffic or the driving environment."

A figure with the lever positions with the "comfortable", "neutral", and "uncomfortable" position was shown to participants during the explanation of the lever positions. This part of the instruction was followed by a short training in which the experimenter instructed the 
participant to imagine a positive, a negative, and a neutral event and use the lever to rate his or her feelings during this event. The participant was then reminded to focus their gaze on the driving situations and not on the lever and the first block of driving situations was started.

\subsection{Comfort rating lever}

Participants rated their subjective experience of the vehicle to vehicle distance on a bidirectional haptic lever (Figure 2). The lever used in this study is an adapted version of the "Vehrs-Hebel” (engl. "Vehrs-Lever"), developed by Wolfgang Vehrs (1986) for the nonverbal rating of stimuli. The self-centering lever-arm protrudes out of the top end of a heavy base that houses the mechanics of the lever. Using an orthogonally placed handle at the top, the lever can be moved within $15 \mathrm{~cm}$, i.e., for $7.5 \mathrm{~cm}$ from its middle position to each edge of the box. Placed under the driving simulator cabin, the lever arm protrudes out of the middle console in front of the gearstick. Tests on the use of the lever for ratings of subjective experiences by Vehrs (1986) as well as a pretest by the authors of this study suggest that participants are able to express their subjective experience accurately with the help of the lever. The lever position is saved as a percentage value with a frequency of $20 \mathrm{~Hz}$. A "comfortable" lever rating, i.e., a participant pulls the lever as close toward him- or herself as possible, results in a $100 \%$ value. A "neutral" lever rating, where the lever is positioned in the middle, results in a 50\% value. An "uncomfortable" rating where a participant pushes the lever as far away as possible from him- or herself results in a $0 \%$ value (Figure 2).

The direction of valence of lever ratings (see 2.4) was chosen for two reasons. First, it is more natural to have "uncomfortable" ratings defined as a lever push away from the body, and "comfortable" ratings as a pulling movement towards the own body (Chen \& Bargh, 1999;

Solarz, 1960). Second, since time headways for a given speed are represented as gaps between the participant's vehicle and the lead vehicle, the lever movement could in theory just copy this gap between the two vehicles. In this case, the lever would present the position of the lead vehicle. Defining the lever ratings in a way that prohibits this replication of the lead vehicle position with the lever helps to prevent this effect. Apart from the exact lever position, the median of all lever ratings is an indicator if the majority of participants rates a distance as comfortable (median position $>50 \%$ ) or uncomfortable (median position $<50 \%$ ). 


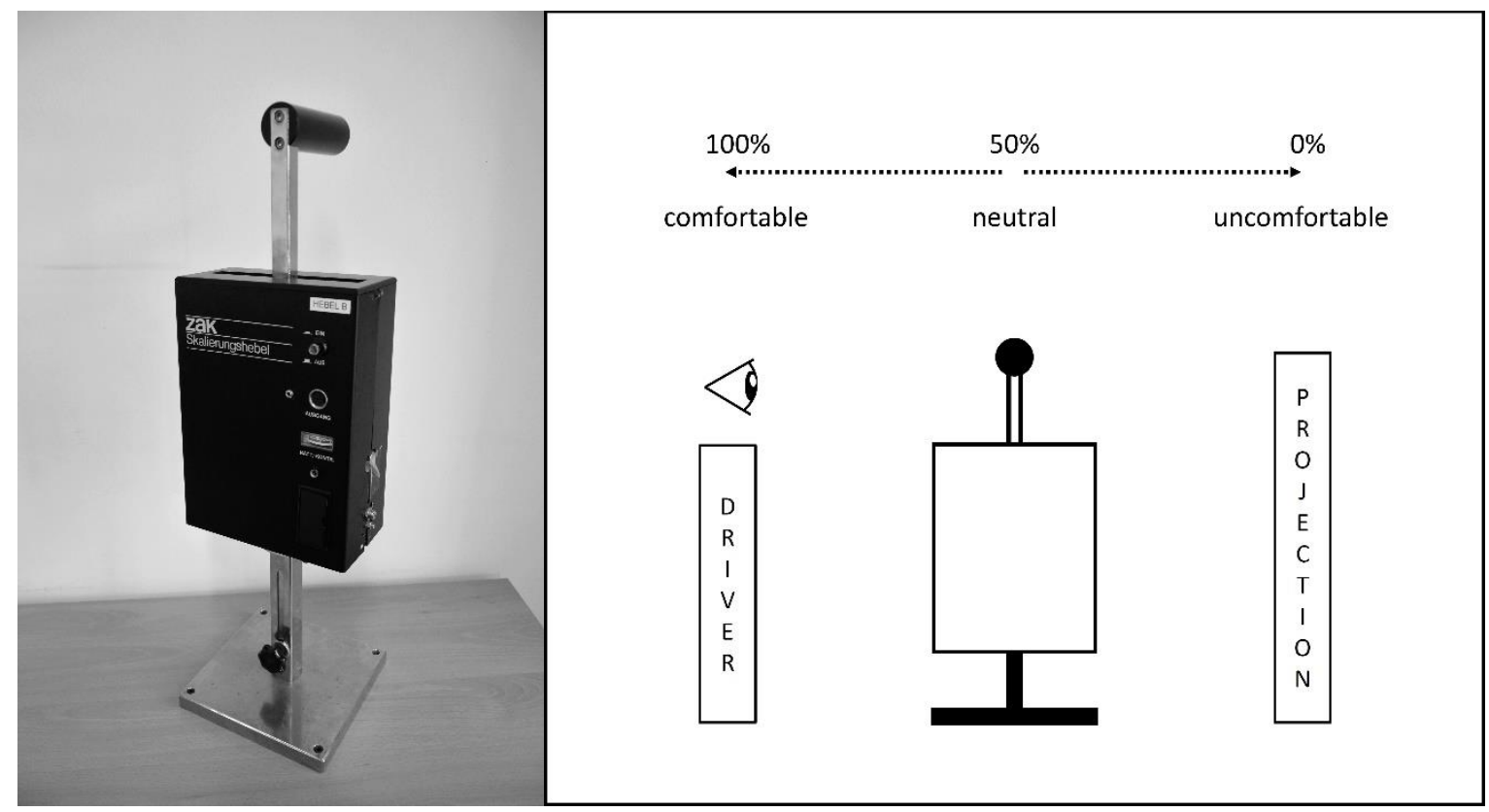

Figure 2. Rating lever and scaling direction.

\subsection{Analysis}

The raw data output of the lever were pre-processed before any calculations were conducted. Since conditions were presented consecutively, the initial lever position of a condition was influenced by the final lever position of the preceding condition. As participants were instructed to maintain a lever position once the lever was at the intended position, we looked at the standard deviation of the lever position, as it indicates movement of the lever.

The standard deviation of all ratings in this study was plotted, including each condition and each participant, resulting in one average of standard deviation for 10 seconds of rating. These 10 seconds were consecutively shortened in 0.5 second steps starting from the beginning, until there were only the last 0.5 seconds of the condition left. The resulting data (Figure 3 ) showed that standard deviation in the lever data decreases as the first few seconds of each condition are eliminated. From Figure 3 it can be assumed that the majority of participants require about 5 seconds to arrive at the intended lever position. Due to this, the lever data of the first five seconds of each condition was not included in the calculation of the lever position. Only the last 5 seconds (100 data points) of each condition are averaged and used as the comfort rating for a given condition. 


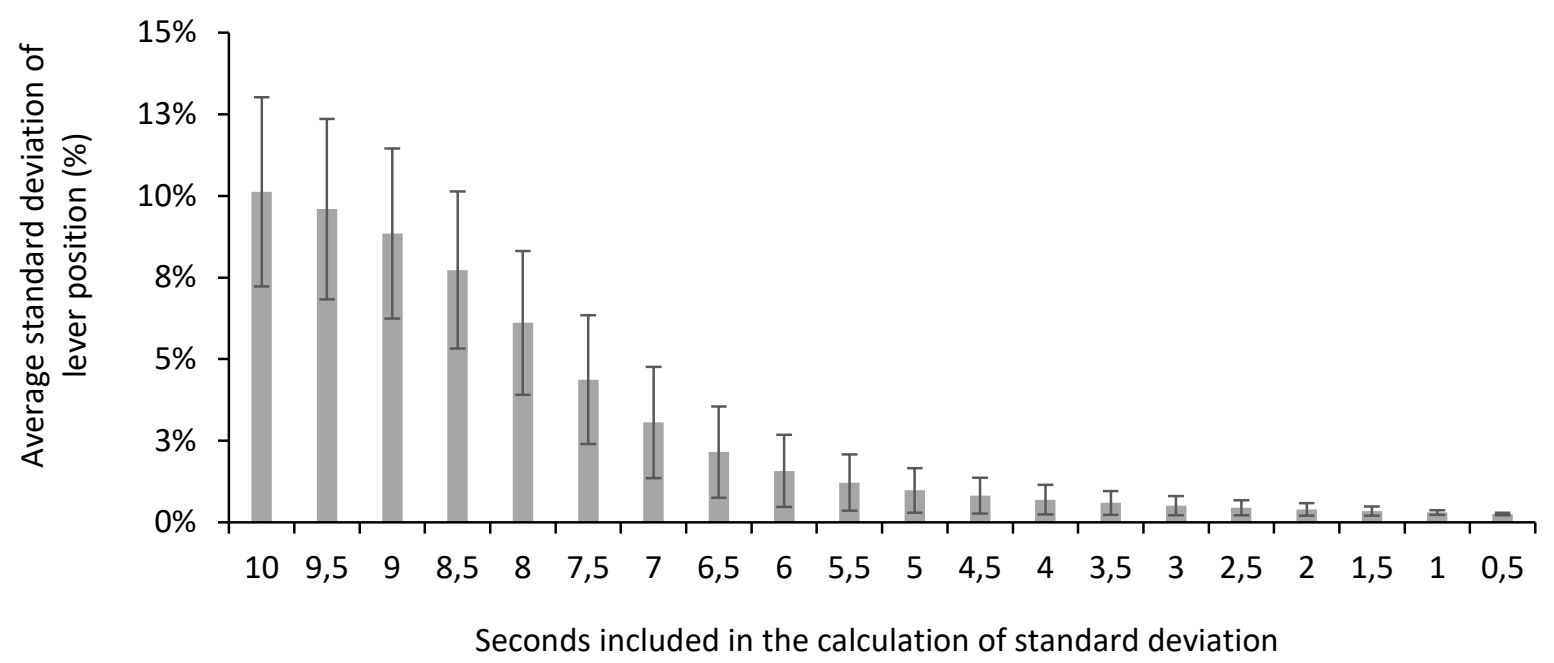

Figure 3. Average standard deviation of the lever position for different condition times, reduced by 0.5 second increments from the start of the condition (error bars show the standard deviation).

All rating data were analyzed by a three-way $(3 \times 3 \times 10)$ repeated measures analysis of variance (ANOVA), with visibility (within-subjects; clear vs. fog vs. truck), speed (within-subjects; $50 \mathrm{~km} / \mathrm{h}$ vs. $100 \mathrm{~km} / \mathrm{h}$ vs. $150 \mathrm{~km} / \mathrm{h}$ ), and time headway (within-subjects; 0.5 vs. 1.0 vs. 1.25 vs. 1.5 vs. 1.75 vs. 2.0 vs. 2.5 vs. 3.0 vs. 3.5 vs. 4.0 ) as the independent variables and comfort (rated through the lever) as the dependent variable.

3. Results

\subsection{Influence of speed on comfortable time headways}

Mean comfort ratings for time headways under different visibility and speed conditions are presented in Figure 4. We assumed that speed does influence comfort ratings for specific time headways. The influence of speed on comfort ratings was tested in a three-way ANOVA, comparing speed as one of the factors at 50, 100 and $150 \mathrm{~km} / \mathrm{h}$. Since Mauchly's Test revealed that the assumption of sphericity had been violated for the main effect of speed $\left(\chi^{2}(2)\right.$ $=8.92, p<.012)$, Greenhouse-Geisser corrected degrees of freedom were used $(\varepsilon=.81)$. There was a significant main effect of speed on comfort ratings of time headways $\left(F_{(1.62,54.98)}\right.$ $\left.=42.22, p<.01, \eta_{p}{ }^{2}=.55\right)$. For nearly all time headways, participants rated following at lower speeds as less comfortable than following at higher speeds (Figure 4). Comfort in the clear visibility condition (top of Figure 4) was lowest for the $50 \mathrm{~km} / \mathrm{h}$ condition, followed by the $100 \mathrm{~km} / \mathrm{h}$ condition, with the $150 \mathrm{~km} / \mathrm{h}$ condition rated as the most comfortable on average. This difference in ratings can also be observed for the fog and the truck condition, where time 
headways of lower speeds are rated as less comfortable when compared to the same time headways at higher speeds. Post-hoc tests using Bonferroni correction for multiple comparisons revealed significant differences between comfort ratings of all three speed conditions (all $p<.01)$.

338

339
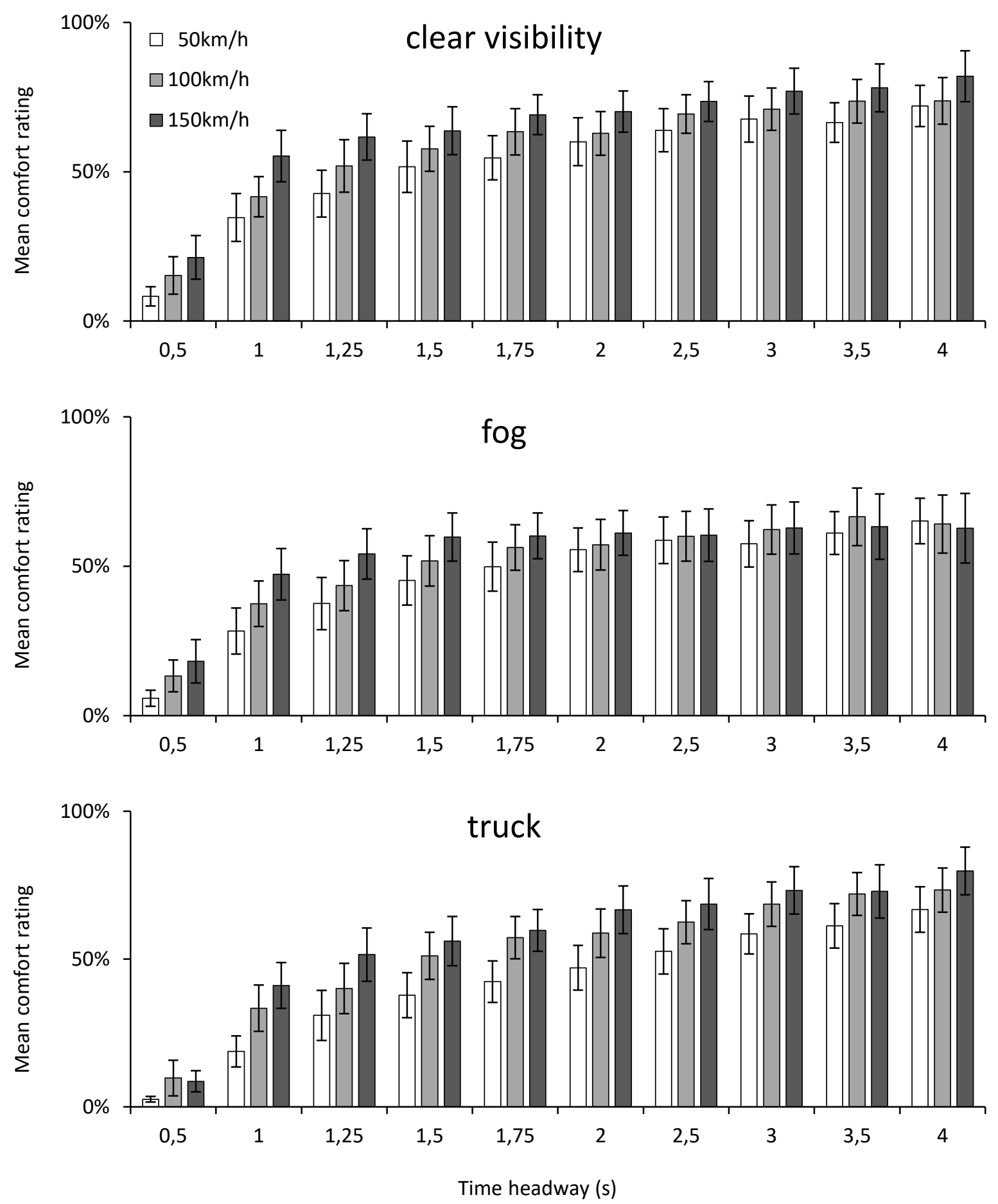

342 Figure 4. Mean comfort ratings for different time headways at 50, 100, and $150 \mathrm{~km} / \mathrm{h}$ and three visibility conditions (error bars show the $95 \%$ confidence interval). 


\subsection{Influence of visibility on comfortable time headways}

345

346

347

348

349

350

351

352

353

354

355

356

357

358

359

360

361

362

363

364

We hypothesized that reduced visibility leads to a decrease in comfort ratings of time headways when compared to clear visibility. To test the influence of visibility on comfort ratings, three visibility conditions (clear vs. fog vs. truck) were compared as one factor in a three-way ANOVA. There was a significant main effect of visibility on comfort ratings of time headways $\left(F_{(2,68)}=16.87, p<.01, \eta_{p}{ }^{2}=.33\right)$. Post-hoc tests using Bonferroni correction for multiple comparisons revealed that comfort in the clear visibility condition is significantly higher than in the truck and the fog condition (both $p<.01$ ). There was no significant difference between comfort ratings of the truck and the fog condition $(p=1.0)$.

Descriptively, participants rated shorter time headways $(<3 s)$ in the fog condition as more comfortable than in the truck condition. However, for larger time headways ( $\geq 3 \mathrm{~s}$ ) following in fog was rated as less comfortable than following a truck. Furthermore, comfort ratings for the clear and truck visibility conditions increase with increasing time headways, while comfort ratings for the fog condition remain more constant even when time headway increases. Due to this effect, large time headways are less comfortable in a foggy environment than in the truck or clear visibility condition.

\subsection{Interaction of visibility and speed}

The ANOVA revealed a significant interaction for the influence of visibility and speed on comfort ratings $\left(F_{(4,136)}=2.86, p=.026, \eta_{p}{ }^{2}=.078\right)$. An interaction graph with a shortened yaxis for better visibility is plotted in Figure 5. 


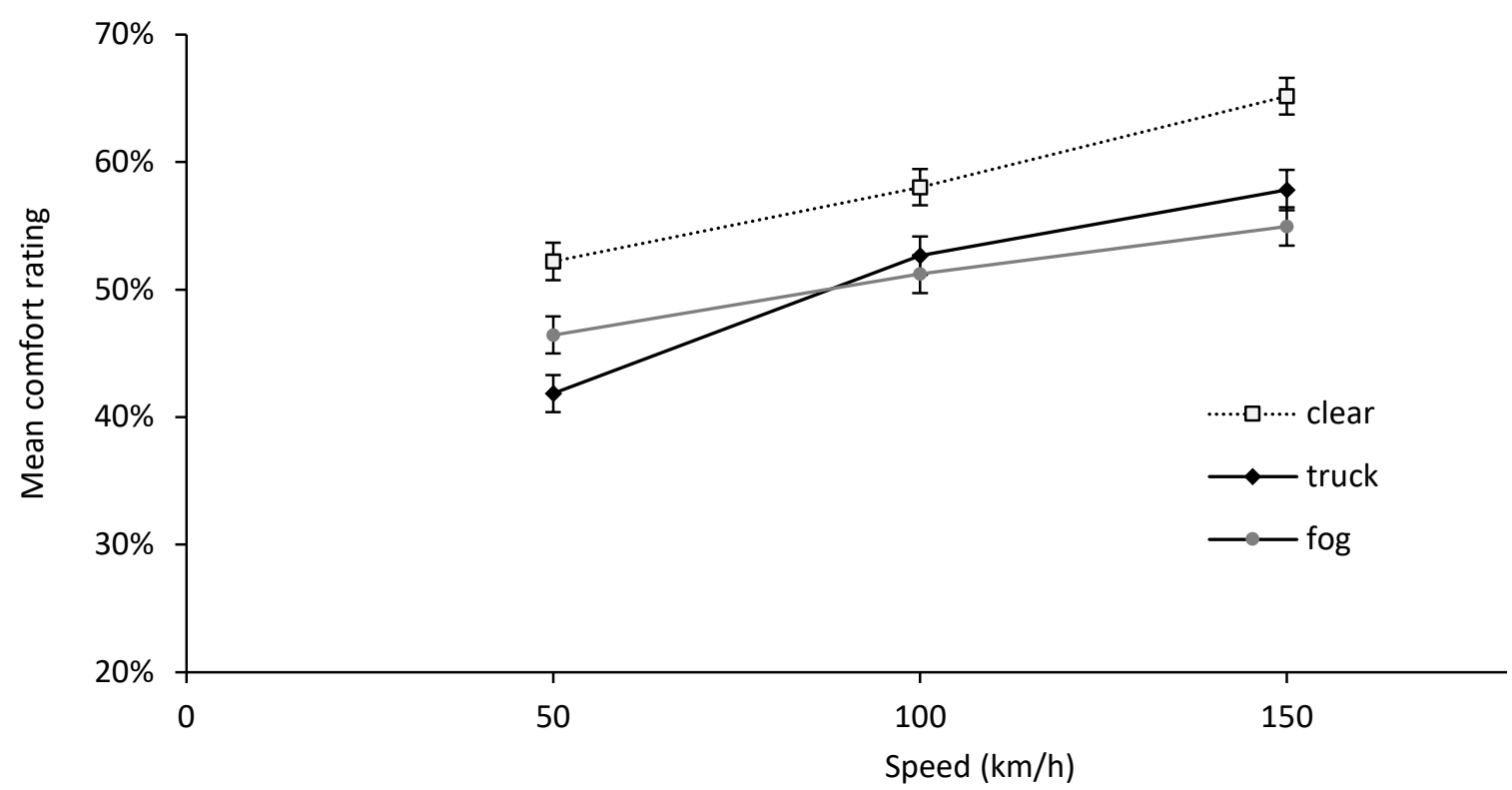

Figure 5. Interaction graph for mean lever ratings for all visibility and speed conditions (please note the shortened y-axis). Error bars show the 95\% confidence interval.

In Figure 5 the main effect of speed is visible, comfort generally increases with higher speeds. A difference between the clear condition and reduced visibility conditions can also be observed, reduced visibility leads to a decrease in comfort, when compared to the clear visibility condition (all $p<.01$ after Bonferroni correction for multiple comparisons). Between the two reduced visibility conditions however, an interaction between visibility and speed can be observed descriptively. For the 100 and $150 \mathrm{~km} / \mathrm{h}$ condition, comfort ratings of the truck and fog condition are descriptively similar and do not significantly differ (all $p>.05)$. However, the mean comfort ratings of the truck condition at $50 \mathrm{~km} / \mathrm{h}$ are descriptively lower than ratings for the fog condition of the same speed (Figure 5). Calculating a separate repeated-measure ANOVA for the $50 \mathrm{~km} / \mathrm{h}$ condition however does not show a significant difference between the two reduced visibility conditions, as it just fails to be significant at $p=0.57$ after Bonferroni correction for multiple comparisons.

\subsection{Comfortable vs. uncomfortable time headways}

Through descriptively analyzing the median lever position for an individual experimental condition, it is possible to determine if a majority of participants rated a given time headway as comfortable or uncomfortable. Therefore, median lever ratings can be used to descriptively 
quantify the influence of speed and visibility changes on comfort ratings of time headways. Median lever positions for all conditions are presented in Table 1.

For example, in the clear condition at $50 \mathrm{~km} / \mathrm{h}$, the majority of participants rate time headways of 1.5 seconds and higher as comfortable, i.e. the median lever rating for these time headways is higher than $50 \%$ indicating comfortable distances (Figure 2). For $100 \mathrm{~km} / \mathrm{h}$ this threshold shifts to 1.25 seconds, i.e. with a speed increase of $50 \mathrm{~km} / \mathrm{h}$ the time headway distance can be reduced by 0.25 seconds without the majority of participants perceiving the distance as uncomfortable. With an additional increase of the speed to $150 \mathrm{~km} / \mathrm{h}$ the time headway distance again can be reduced by 0.25 seconds, resulting in a following distance of 1.0 seconds that is still perceived as comfortable by a majority of participants. For reduced visibility conditions, i.e. driving in fog or behind a truck, a similar effect of speed can be found. With increasing speed, time headway following distances can be decreased without the majority of participants perceiving the distances as uncomfortable (see Table 1).

For a reduced visibility road environment, it is necessary to increase time headway. At $50 \mathrm{~km} / \mathrm{h}$, time headway needs to be increased by 1 second when a driver is transferring from e.g. a clear visibility environment, to a foggy road environment, or the lead car changes from a passenger car to a truck. At higher speeds, this shift is less pronounced but still present (Table 1). Through Table 1 it is possible to exactly quantify how time headways need to be changed for varying speeds and visibility.

.

.

(1)

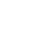


411 Table 1. Median lever ratings for different time headways (TH), speeds, and visibility 412 conditions.

\begin{tabular}{|c|c|c|c|c|c|c|c|c|c|}
\hline \multirow[b]{2}{*}{$\mathrm{TH}$} & \multicolumn{3}{|c|}{$50 \mathrm{~km} / \mathrm{h}$} & \multicolumn{3}{|c|}{$100 \mathrm{~km} / \mathrm{h}$} & \multicolumn{3}{|c|}{$150 \mathrm{~km} / \mathrm{h}$} \\
\hline & clear & fog & truck & clear & fog & truck & clear & fog & truck \\
\hline 0.5 & $2.8 \%$ & $1.9 \%$ & $1.6 \%$ & $9.2 \%$ & $6.1 \%$ & $2.4 \%$ & $14.8 \%$ & $12.0 \%$ & $3.5 \%$ \\
\hline 1.0 & $29.4 \%$ & $23.9 \%$ & $17.2 \%$ & $42.4 \%$ & $35.1 \%$ & $25.6 \%$ & $51.4 \%$ & $43.8 \%$ & $38.2 \%$ \\
\hline 1.25 & $38.8 \%$ & $34.6 \%$ & $24.6 \%$ & $51.5 \%$ & $40.6 \%$ & $34.7 \%$ & $56.2 \%$ & $51.7 \%$ & $45.9 \%$ \\
\hline 1.5 & $51.0 \%$ & $42.5 \%$ & $35.5 \%$ & $52.0 \%$ & $44.6 \%$ & $43.7 \%$ & $58.6 \%$ & $60.2 \%$ & $51.5 \%$ \\
\hline 1.75 & $51.5 \%$ & $44.6 \%$ & $39.4 \%$ & $56.2 \%$ & $51.4 \%$ & $51.5 \%$ & $69.1 \%$ & $60.4 \%$ & $52.4 \%$ \\
\hline 2.0 & $52.2 \%$ & $49.9 \%$ & $44.6 \%$ & $58.0 \%$ & $51.5 \%$ & $52.4 \%$ & $66.6 \%$ & $56.3 \%$ & $63.4 \%$ \\
\hline 2.5 & $60.3 \%$ & $52.1 \%$ & $51.9 \%$ & $66.1 \%$ & $57.0 \%$ & $52.0 \%$ & $75.7 \%$ & $59.5 \%$ & $69.1 \%$ \\
\hline 3.0 & $65.0 \%$ & $51.6 \%$ & $57.1 \%$ & $67.6 \%$ & $59.4 \%$ & $62.7 \%$ & $83.7 \%$ & $64.5 \%$ & $74.6 \%$ \\
\hline 3.5 & $66.4 \%$ & $55.8 \%$ & $56.9 \%$ & $75.9 \%$ & $68.7 \%$ & $70.9 \%$ & $88.1 \%$ & $56.4 \%$ & $81.4 \%$ \\
\hline 4.0 & $72.5 \%$ & $61.3 \%$ & $65.9 \%$ & $72.8 \%$ & $68.3 \%$ & $75.2 \%$ & $97.0 \%$ & $62.4 \%$ & $96.4 \%$ \\
\hline
\end{tabular}

Comfortable ratings with median lever position $>50 \%$ in bold.

\section{Discussion}

In this study we examined the influence of different time headways on subjective comfort when following another vehicle with different speeds under different visibility conditions in a highly automated vehicle. In our first hypothesis we postulated that speed would influence the subjective comfort for a given time headway. Our data supports this hypothesis, time headways at lower speeds were rated as less comfortable than the same time headways at higher speeds. This result stands in contrast to results of earlier studies on self- and assisteddriving, where the subjective experience of a given time headway was not influenced by speed (Siebert et al., 2014, 2017). The assumption of equal comfort for identical time headways can therefore not be extended to highly automated driving. It is important to keep in mind that this study differs from earlier studies on time headway and subjective experience, in 
that the simulated car in this study was highly automated. In contrast to earlier studies (see Section 1.1.), participants did not have any control over the car, which could have a general effect on perceived comfort levels for time headways. If there was a simple effect of control, i.e. that less control (as in highly automated driving) leads to less comfort for a given time headway, this effect would be constant for different speeds. This simple effect would therefore not lead to the results found in this study. Our analysis of median lever ratings reveals that with an increase in speed of $50 \mathrm{~km} / \mathrm{h}$, time headway distances can be reduced by 0.25 seconds without the majority of participants perceiving the distance as uncomfortable. Although the relative validity of driving simulators has been established for speed and vehicle to vehicle distances (Godley, Triggs, \& Fildes, 2002; Risto, \& Martens, 2014), the exact time headway distances found in this study might not be directly transferable to real life driving. Nonetheless, our results indicate that time headways in highly automated driving will need to be adaptive to speed.

In our second hypothesis we postulated that reduced visibility leads to a decrease in comfort ratings when compared to the same distances in the clear visibility condition. In this study, participants rated time headways as significantly less comfortable when visibility was reduced by a truck or due to fog, supporting our hypothesis. Our analysis of median comfort ratings shows that reduced visibility requires an increase in up to 1 second time headway, to maintain a comfortable rating of the distance by a majority of the participants. As discussed earlier, research on self-driving has not found a consistent effect of reduced visibility on car following behavior. The results of this study appear to support findings of increased headway following in reduced visibility conditions, and expand these findings to highly automated driving.

While there was no significant difference of comfort ratings between the fog and the truck condition, there was a significant interaction of visibility and speed. Although the effect just failed to be significant in posthoc testing, following a truck was descriptively rated as less comfortable than following in fog in the $50 \mathrm{~km} / \mathrm{h}$ condition. This descriptive effect was not present in the 100 or $150 \mathrm{~km} / \mathrm{h}$ condition.

A descriptive effect of fog on comfort ratings of time headways can be observed for larger time headways. While comfort increases with time headways in the truck condition, comfort ratings stay more constant in the fog condition, although time headway distances are increasing. This effect is most pronounced in the $150 \mathrm{~km} / \mathrm{h}$ condition. A possible explanation for this effect is the visibility limit of 200 meters set for the fog condition in this study. 
Although even in the largest time headway conditions of four seconds the lead vehicle is always visible (as the largest distance of the $150 \mathrm{~km} / \mathrm{h}$ condition is 166.66 meters), the lead vehicle is close to the edge of the visible driving environment. While this does not directly influence the following car, participants might anticipate a potential loss of visibility of the lead vehicle. This might be the onset of the effect of close following to keep eye-contact to the lead vehicle, found by Broughton et al. (2007). The influence of the visibility range of driving in fog needs to be researched further to be able to interpret the influence of this effect.

This study has multiple limitations. The simulation of driving in a fixed based simulator, and especially the simulation of fog is different from real life driving and reduced visibility in the real-life driving environment. The inconclusive results of earlier studies on the influence of fog on following distances (Broughton, Switzer, and Scott, 2007; Saffarian, Happee, De Winter, 2012; Van der Hulst, Rothengatter, Meijman, 1998) could in part be attributed to differences in the display of fog in driving simulators. The results therefore have to be confirmed in real life driving conditions. Although the truck condition was introduced to restrict the forward visibility of participants, the truck model differed from vehicle models in the fog and clear condition due to its larger size. Vehicle size has been found to influence following behaviour (Brackstone, Waterson, \& McDonald, 2009; Duan, Li, \& Salvendy, 2013; Green \& Yoo, 1999; Sayer, Mefford, \& Huang, 2000). As such, effects found in the truck condition cannot be solely attributed to the obstructed forward view, but could further be influenced by the larger vehicle size. Future experiments should take this into account, e.g. by using a lead vehicle model of normal size with opaque windows.

The highly automated vehicle that was simulated in this study was considerably simplified. The car drove with a constant speed of 50,100 , or $150 \mathrm{~km} / \mathrm{h}$, kept the lane perfectly, and never overtook another vehicle. Future studies need to simulate highly automated vehicles that are closer to their real life counterparts in their behavior. The exposure to highly automated driving was very limited for most participants, it can be assumed that none of them had used a highly automated vehicle in the past. It seems advisable to give participants more time to familiarize themselves with the behavior of the simulated car as drivers need time to develop a mental model of a car's automation (Beggiato, Pereira, Petzoldt, Krems, 2015). Apart from little experience with highly automated driving, participants in this study were relatively young, with a mean age of only 22.5 years, resulting in a relatively short driving experience. Hence, future studies should aim to have a more representative sample. 
In contrast to earlier studies on the topic of time headway and vehicle automation (Siebert et al., 2014, 2017) there was no self-driving condition in this study, where the driver has complete control over the vehicle. Implementing a self-driving condition in this study within the experimental framework of comfort rated on a scaling lever would not have been possible, since drivers need both hands to control the vehicle in self-driving, i.e. drivers cannot rate their comfort through the lever while driving. Since it is unclear how comfort of time headways in self- and highly automated driving relate to each other, future studies should include a self-driving condition, even if it uses a different methodology for the collection of comfort data. Further, despite earlier studies on the use of haptic rating devices (Charlton, Starkey, Perrone, \& Isler, 2014; Vehrs, 1986) for the subjective experience of study participants, the novel use of a rating lever in traffic psychological experiments necessitates the replication of our results with established methodological approaches. Despite these limitations, this study provides a basis for the further investigation of additional variables that influence following distances in highly automated driving.

In summary, the results of this study add to the existing literature on car following and are a first step in expanding the field of research on car following from self-driving to highly automated driving. Speed influenced the comfort ratings of time headways, a finding that contrasts with results found in self and assisted driving. Reduced visibility led to a decrease in comfort. Results indicate that time headways in highly automated driving will need to be adaptively adjusted to speed and the road environment. Future studies need to investigate these effects in real life driving, and investigate the influence of differences in visibility range during fog in more detail. .

.

(4)

(1)

.




\section{References}

Al-Ghamdi, A. S. (2007). Experimental evaluation of fog warning system. Accident Analysis \& Prevention, 39(6), 1065-1072.

Ayres, T. J., Li, L., Schleuning, D., \& Young, D. (2001). Preferred time-headway of highway drivers. In Intelligent Transportation Systems, 2001. Proceedings. 2001 IEEE (pp. 826-829). IEEE.

Beggiato, M., Pereira, M., Petzoldt, T., \& Krems, J. (2015). Learning and development of trust, acceptance and the mental model of ACC. A longitudinal on-road study. Transportation research part F: traffic psychology and behaviour, 35, 75-84.

Bellem, H., Schönenberg, T., Krems, J. F., \& Schrauf, M. (2016). Objective metrics of comfort: developing a driving style for highly automated vehicles. Transportation research part F: traffic psychology and behaviour, 41, 45-54.

Brackstone, M., Waterson, B., \& McDonald, M. (2009). Determinants of following headway in congested traffic. Transportation Research Part F: Traffic Psychology and Behaviour, 12(2), 131-142.

Broughton, K. L., Switzer, F., \& Scott, D. (2007). Car following decisions under three visibility conditions and two speeds tested with a driving simulator. Accident Analysis \& Prevention, 39(1), 106-116.

Charlton, S. G., Starkey, N. J., Perrone, J. A., \& Isler, R. B. (2014). What's the risk? A comparison of actual and perceived driving risk. Transportation research part $F$ : traffic psychology and behaviour, 25, 50-64.

Chen, M., \& Bargh, J. A. (1999). Consequences of automatic evaluation: Immediate behavioral predispositions to approach or avoid the stimulus. Personality and social psychology bulletin, 25(2), 215-224.

De Vos, A. P., Theeuwes, J., Hoekstra, W., \& Coëmet, M. J. (1997). Behavioral aspects of automatic vehicle guidance: Relationship between headway and driver comfort. Transportation research record, 1573(1), 17-22. 
De Waard, D., van der Hulst, M., Hoedemaeker, M., \& Brookhuis, K. A. (1999). Driver behavior in an emergency situation in the Automated Highway System. Transportation human factors, 1(1), 67-82.

Duan, J., Li, Z., \& Salvendy, G. (2013). Risk illusions in car following: Is a smaller headway always perceived as more dangerous?. Safety science, 53, 25-33.

Godley, S. T., Triggs, T. J., \& Fildes, B. N. (2002). Driving simulator validation for speed research. Accident analysis \& prevention, 34(5), 589-600.

Green, P., \& Yoo, H. (1999). Driver behavior while following cars, trucks, and buses (No. UMTRI-99-14).

Lewis-Evans, B., De Waard, D., \& Brookhuis, K. A. (2010). That's close enough-A threshold effect of time headway on the experience of risk, task difficulty, effort, and comfort. Accident Analysis \& Prevention, 42(6), 1926-1933.

Risto, M., \& Martens, M. H. (2014). Driver headway choice: A comparison between driving simulator and real-road driving. Transportation research part F: traffic psychology and behaviour, 25, 1-9.

Saffarian, M., Happee, R., \& De Winter, J. (2012). Why do drivers maintain short headways in fog? A driving-simulator study evaluating feeling of risk and lateral control during automated and manual car following. Ergonomics, 55(9), 971-985.

Sayer, J. R., Mefford, M. L., \& Huang, R. (2000). The effect of lead-vehicle size on driver following behavior. Ann Arbor, 1001, 48109-2150.

Siebert, F. W., Oehl, M., Bersch, F., \& Pfister, H. R. (2017). The exact determination of subjective risk and comfort thresholds in car following. Transportation research part $F$ : traffic psychology and behaviour, 46, 1-13.

Siebert, F. W., Oehl, M., \& Pfister, H. R. (2014). The influence of time headway on subjective driver states in adaptive cruise control. Transportation research part $F$ : traffic psychology and behaviour, 25, 65-73.

Solarz, A. K. (1960). Latency of instrumental responses as a function of compatibility with the meaning of eliciting verbal signs. Journal of experimental psychology, 59(4), 239. 
575 Taieb-Maimon, M., \& Shinar, D. (2001). Minimum and comfortable driving headways:

576 Reality versus perception. Human Factors: The Journal of the Human Factors and

577 Ergonomics Society, 43(1), 159-172.

578 Telpaz, A., Baltaxe, M., Hecht, R. M., Cohen-Lazry, G., Degani, A., \& Kamhi, G. (2018). An

579 Approach for Measurement of Passenger Comfort: Real-Time Classification based on In-

580 Cabin and Exterior Data. In 2018 21st International Conference on Intelligent Transportation

581 Systems (ITSC) (pp. 223-229). IEEE.

582 Van der Hulst, M., Rothengatter, T., \& Meijman, T. (1998). Strategic adaptations to lack of 583 preview in driving. Transportation research part F: traffic psychology and behaviour, 1(1), $58459-75$.

585 Van Winsum, W. \& Heino, A. (1996). Choice of time-headway in car-following and the role 586 of time-to-collision information in braking. Ergonomics, 39(4), 579-592.

587 Van Winsum, W. (1999). The human element in car following models. Transportation 588 research part F: traffic psychology and behaviour, 2(4), 207-211.

589 Vehrs, W. (1986). Nicht-verbale Erlebnisbeschreibung. Göttingen: Hogrefe.

590 Wasielewski, P. (1981). The effect of car size on headways in freely flowing freeway traffic.

591 Transportation Science, 15(4), 364-378. 